\title{
Wave Dispersion, Growth Rates, and Mode Converter Analysis for a Sheet Beam, Hybrid-Mode Cerenkov Amplifier
}

\author{
Sheng-Fuh R. Chang, John E. Scharer, and John H. Booske, Member, IEEE
}

\begin{abstract}
The hybrid-mode dispersion relation and resonant growth rates are solved for a finite-thickness sheet electron beam propagating through a rectangular guide with a thin dielectric slab. Analytic results for the growth rates, bandwidth, and mode competition for an infinite magnetic field in the limit of a dilute beam are then presented. To properly couple to the desired $\mathrm{EH}_{10}$ hybrid amplifier slow wave mode, the coupled waveguide mode equations are solved for a dielectric taper to accomplish the $\mathrm{TE}_{10}$ to $\mathrm{EH}_{10}$ mode transition. A piecewise linear taper which suppresses the competing $\mathrm{EH}_{11}$ mode and other hybrid modes is developed and the overall amplifier system is discussed.
\end{abstract}

\section{INTRODUCTION}

$\mathbf{T}$ THE most common electron optics configuration for microwave slow-wave (or Cerenkov) amplifiers includes a round cross-section (or pencil) beam, an electrostatically focused Pierce gun, and confined flow with either an axial magnetic field or PPM focusing. The most constraining of these features for high-power operation at millimeter wavelengths is the choice of a round-beam cross section. High power implies keeping the beam current high, while shrinking the beam diameter to accommodate the smaller clearances associated with millimeter-wave (MMW) circuit structures. The subsequent increase in beam space charge makes beam confinement difficult and is responsible for high body currents. Consequently, one rapidly encounters a practical maximum limit on either operating frequency (for a given average power requirement) or output RF average power (for a given frequency requirement). This design limit for pencil beam devices is especially relevant to "low-voltage," "soft" electron beams (i.e., less than $100 \mathrm{kV}$.)

One solution to this challenge that has recently received increased scrutiny is to use "sheet" electron beams [1]-[6]. By sheet electron beam, we refer here to beams with rectangular cross section having transverse dimension aspect ratios greater than or equal to 10 . Historically, this configuration has been plagued by concerns of diocotron [7]-[10] or similar velocityshear driven beam instabilities. However, recent experiments indicate that stable sheet beam transport is possible by using

Manuscript received August 30,1991; revised January 28, 1992. This work was supported in part by the University of Wisconsin-Madison Graduate School and NSF Presidential Young Investigator Award ECS-9057675, and by the U.S. Air Force through Grant AFOSR-91-0381.

The authors are with the Department of Electrical and Computer Engineering, University of Wisconsin-Madison Madison, Wisconsin 53706.

IEEE Log Number 9108112. either periodic [1], [2] or sufficiently strong axial magnetic field focusing [4].

The advantage of sheet electron beams is that while one transverse dimension remains small and compatible with MMW circuit clearances, the other transverse dimension is enlarged to reduce beam space charge. In this manner, high beam current and higher average RF power operation are possible in a single device. From this perspective, it is desirable to increase the beam's wide transverse dimension virtually without limit. Realistically, input RF coupling as well as beam and cathode alignment tolerances become more problematic as the sheet beam's design width is increased.

This paper analyzes several of the physically important features for a MMW, Cerenkov sheet beam amplifier with a configuration which is readily adaptable to rectangular waveguide input/output couplers - i.e., a modest-width sheet beam with transverse dimension ratios between 10 and 20 . One of the most important consequences of using finite-width sheet beams is that this implies a finite-width rectangular Cerenkov structure, rather than the infinitely wide parallel-plate configuration of [5], [11]-[14]. As a result, the interaction involves "hybrid" modes [15] rather than pure TE or TM modes. We have incorporated this feature, as well as finite beam thickness and spacing from the slow-wave structures into our dispersion and growth-rate analyses of Section II. Furthermore, consistent with our interest in an amplifier device, we have evaluated requirements for efficient, low reflection, input and output mode conversion tapers. These converters permit the input and output waves to be the conventional $\mathrm{TE}_{10}$ mode, launched and radiated from an ordinary smooth-wall rectangular guide. This hybrid mode converter analysis is presented in Section III. Finally, in Section IV, we comment on other relevant issues, including sheet beam stability, as well as limits to the validity of our model computations.

\section{Dispersion RELATION AND RESONANT GROWTH RATE}

\section{A. Modeling of a Confined Electron Beam}

The dielectric Cerenkov maser consists of a relativistic sheet electron beam streaming through a dielectric-lined rectangular guide as shown in Fig. 1. An infinite magnetostatic field is assumed so that the effect of electron gyromotion is excluded and only the space-charge wave of the beam is considered. The thermal effect of the beam is neglected so that the sheet 


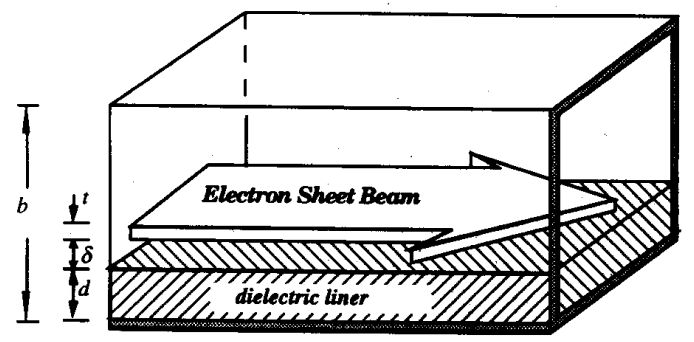

(a)

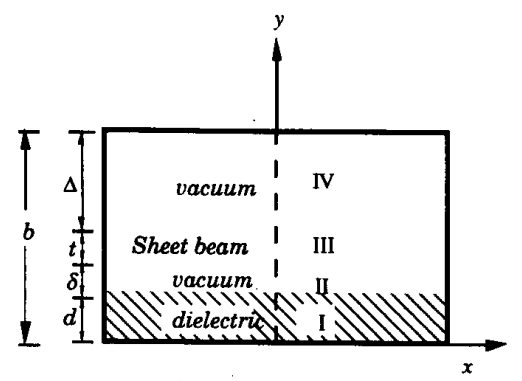

(b)

Fig. 1. Schematic sketch of a dielectric-lined guide with a sheet beam streaming through it. (a) Three-dimensional view. (b) Cross-sectional view.

beam can be treated as a cold fluid. Under these assumptions, the electron's motion is constrained to the axial dimension and is perturbed by the electromagnetic wave, considered here as a first-order perturbation. By linearizing the Lorentz force equation and the Maxwell equations, the beam can be replaced by an effective dielectric constant [14]

$$
\overline{\bar{\epsilon}}=\epsilon_{0}\left[\begin{array}{ccc}
1 & 0 & 0 \\
0 & 1 & 0 \\
0 & 0 & 1+\chi_{b}
\end{array}\right]
$$

where the susceptibility $\chi_{b} \equiv-\omega_{p}^{2} / \gamma_{0}^{3}\left(\omega-k_{z} v_{0}\right)^{2}$ reflects the electron polarization response to the perturbing $E_{z}$ field.

A beam propagating through a finite magnetic guiding field can excite the fast and slow space-charge waves as well as the fast and slow cyclotron waves. The slow space-charge mode can become unstable when the synchronism holds. Case et al. [16] and Kuo et al. [17] examined the competition of space-charge-Cerenkov and cyclotron-Cerenkov instabilities for an ideal case of beam propagating through a fully filled planar guide. Based on their findings, the cyclotron instability dominates if

$$
\gamma>15\left[\frac{B_{0}^{3}}{J_{b} f_{o} \epsilon_{r}^{4 / 3}}\right]^{3 / 10}
$$

where $B_{0}$ is the axial magnetic field in $\mathrm{kG}, J_{b}$ is the beam current density in $\mathrm{A} / \mathrm{cm}^{2}, f_{o}$ is the operating frequency in $\mathrm{GHz}$, and $\epsilon_{r}$ denotes the relative dielectric constant. For parameters of interest here, $B_{0}=4 \mathrm{kG}, J_{b}=10 \mathrm{~A} / \mathrm{cm}^{2}, \epsilon_{r}=$ 10 , and $f_{o}=35 \mathrm{GHz}$, the cyclotron-Cerenkov interaction dominates for beam voltages larger than $1.5 \mathrm{MV}$, which is far above the beam voltage range $(<100 \mathrm{kV})$ considered in the following analysis.
Freund and Ganguly [18] have used numerical techniques to analyze a dielectric-lined cylindrical wave guide of $3 \mathrm{~mm}$ radius to amplify single-mode $100-\mathrm{GHz}$ radiation. They carry out a nonlinear solution of the problem, neglecting space charge, and find gain and efficiency which agree with an earlier experiment. Garate et al. [5], [6] have derived the small signal gain for the parallel-planar, dielectric Cerenkov configuration in the tenuous beam limit and compared it with experimental results. The $\mathrm{TM}_{01}$ planar mode they examine predicts submillimeter growth rates as high as $3 \mathrm{~dB} / \mathrm{cm}$ for closer beam-dielectric gaps $(0.1 \mathrm{~mm})$ than are considered here and for 50-A currents. In this work, we focus on rectangular guide, hybrid-mode linear growth rates, mode competition, and mode converters necessary to realize this Cerenkov amplifier.

\section{B. An Equivalent Multilayer Field Problem}

Once the susceptibility $\chi_{b}$ is obtained, the problem is reduced to a multilayer field problem. Note that this partially filled waveguide structure cannot support either pure TE or TM modes. Hence, we consider general cases of hybrid modes. In each layer, the relation of field components and the governing wave equation is found by manipulating the Maxwell equations:

For the $E H_{m n}$ Mode:

$$
\begin{gathered}
E_{y}(x, y, z, t)=E_{y}(y) \sin k_{x}\left(x+\frac{a}{2}\right) e^{-i \omega t+i k_{z} z} \\
E_{z}(y)=\frac{i k_{z}}{k_{x}^{2}+k_{z}^{2} S} \frac{\partial E_{y}}{\partial y} \quad H_{x}(y)=\frac{-k_{z}}{k_{x}^{2}+k_{z}^{2} S} \omega \epsilon_{0} \epsilon_{r} E_{y} \\
E_{x}(y)=\frac{k_{x}}{k^{2}+k_{z}^{2} S} \frac{\partial E_{y}}{\partial y} \quad H_{z}(y)=\frac{-i k_{z}}{k_{x}^{2}+k_{z}^{2} S} \omega \epsilon_{0} \epsilon_{r} E_{y} \\
\frac{d^{2}}{d y^{2}} E_{y}+\frac{k_{x}^{2}+k_{z}^{2} S}{k_{x}^{2}+k_{x}^{2}}\left[\frac{\omega^{2}}{c^{2}} \epsilon_{r}-k_{z}^{2}-k_{x}^{2}\right] E_{y}=0
\end{gathered}
$$

For the $H E_{m n}$ Mode:

$$
\begin{gathered}
H_{y}(x, y, z, t)=H_{y}(y) \cos k_{x}\left(x+\frac{a}{2}\right) e^{-i \omega t+i k_{z} z} \\
H_{z}(y)=\frac{i k_{z}}{k_{x}^{2}+k_{z}^{2}} \frac{\partial H_{y}}{\partial y} \quad E_{x}(y)=\frac{-k_{z} S}{k_{x}^{2}+k_{z}^{2} S} \omega \mu H_{y} \\
H_{x}(y)=\frac{-k_{x}}{k_{x}^{2}+k_{z}^{2}} \frac{\partial H_{y}}{\partial y} \quad H_{z}(y)=\frac{-i k_{z}}{k_{x}^{2}+k_{z}^{2} S} \omega \mu H_{y} \\
\frac{d^{2}}{d y^{2}} H_{y}+\frac{k_{x}^{2}+k_{z}^{2}}{k_{x}^{2}+k_{x}^{2} S}\left[\frac{\omega^{2}}{c^{2}} \epsilon_{r} S-k_{z}^{2} S-k_{x}^{2}\right] H_{y}=0
\end{gathered}
$$

with

$$
S=1+\chi_{b} \Theta(y), \quad \Theta(y)= \begin{cases}1, & \text { inside the beam } \\ 0, & \text { elsewhere }\end{cases}
$$

where $\epsilon_{r}=1$ in the vacuum layers, $\epsilon_{r}=1+\chi_{b}$ in the beam layer, and $\epsilon_{r}$ denotes the relative dielectric constant of the material in the dielectric layer.

Solving (2) and (3) and applying the appropriate boundary conditions, we obtain the dispersion equation for the beammode Cerenkov interaction: 
For the $E H_{m n}$ Mode as:

$$
\begin{aligned}
D_{E}\left(\omega, k_{z}\right)= & \alpha_{E}^{2} \kappa_{b}^{2} \tanh \kappa_{b} t\left[\kappa_{d} \tan \kappa_{d} d \tanh \kappa_{0} \delta-\epsilon_{r} \kappa_{0}\right] \\
& +\alpha_{E} \kappa_{0} \kappa_{b}\left[\kappa_{d} \tan \kappa_{d} d-\epsilon_{r} \kappa_{0} \tanh \kappa_{0}(\Delta+\delta)\right] \\
& \cdot\left[1+\tanh \kappa_{0} \Delta \tanh \kappa_{0} \delta\right] \\
& +\kappa_{0}^{2} \tanh \kappa_{b}^{t} \tanh \kappa_{0} \Delta \\
& \cdot\left[\kappa_{d} \tan \kappa_{d} d-\epsilon_{r} \kappa_{0} \tanh \kappa_{0} \delta\right] \\
= & 0
\end{aligned}
$$

For the $H E_{m n}$ Mode as:

$$
\begin{aligned}
D_{H}\left(\omega, k_{z}\right)= & \alpha_{H}^{2} \kappa_{b}^{2} \tanh \kappa_{b} t\left[\kappa_{d} \cot \kappa_{d} d+\kappa_{0} \operatorname{coth} \kappa_{0} \delta\right] \\
& +\alpha_{H} \kappa_{0} \kappa_{b}\left[\kappa_{d} \cot \kappa_{d} d-\kappa_{0} \operatorname{coth} \kappa_{0}(\Delta+\delta)\right] \\
& \cdot\left[\operatorname{coth} \kappa_{0} \delta+\operatorname{coth} \kappa_{0} \Delta\right] \\
& +\kappa_{0}^{2} \tanh \kappa_{b} t \operatorname{coth} \kappa_{0} \Delta \\
& \cdot\left[\kappa_{d} \cot \kappa_{d} d \operatorname{coth} \kappa_{0} \delta+\kappa_{0}\right] \\
= & 0
\end{aligned}
$$

where $\kappa_{d}, \kappa_{0}, \kappa_{b}$ are the transverse wave numbers in the dielectric, vacuum beam, and beam regions, respectively,

$$
\begin{aligned}
\kappa_{0} & =\sqrt{k_{z}^{2}+k_{x}^{2}-\frac{\omega^{2}}{c^{2}}}, \quad \kappa_{d}=\sqrt{\frac{\omega^{2}}{c^{2}} \epsilon_{r}-k_{z}^{2}-k_{x}^{2}} \\
\kappa_{b, E} & =\sqrt{k_{z}^{2}+k_{x}^{2}-\frac{\omega^{2}}{c^{2}}} \sqrt{\frac{k_{x}^{2}+k_{z}^{2} S}{k_{x}^{2}+k_{z}^{2}}} \\
\kappa_{b, H} & =\sqrt{k_{z}^{2} S+k_{x}^{2}-\frac{\omega^{2}}{c^{2}} S} \sqrt{\frac{k_{x}^{2}+k_{z}^{2}}{k_{x}^{2}+k_{z}^{2} S}} \\
\alpha_{E} & =\frac{k_{x}^{2}+k_{z}^{2}}{k_{x}^{2}+k_{z}^{2} S} \quad \alpha_{H}=\frac{k_{x}^{2}+k_{z}^{2} S}{k_{x}^{2} S+k_{z}^{2} S} .
\end{aligned}
$$

The dispersion equations obtained above are suitable for operation in the collective regime (including the high-gain Compton and Raman regimes), where collective space-charge effects are important. Note that by letting the beam thickness $t$ go to zero (no beam case), the dispersion equations reduce to those for a dielectric-lined guide without the beam as follows:

$$
\kappa_{d} \tan \kappa_{d} d=-\epsilon_{r} \kappa_{0} \tan \kappa_{0}(b-d), \quad \text { for the } \mathrm{EH}_{m n}^{\mathrm{fast}} \text { mode }
$$

$\kappa_{d} \tan \kappa_{d} d=\epsilon_{r} \kappa_{0} \tanh \kappa_{0}(b-d), \quad$ for the $\mathrm{EH}_{m n}^{\text {slow }}$ mode

$$
\kappa_{d} \cot \kappa_{d} d=-\kappa_{0} \cot \kappa_{0}(b-d), \quad \text { for the } \mathrm{HE}_{m n}^{\text {fast }} \text { mode }
$$

$\kappa_{d} \cot \kappa_{d} d=-\kappa_{0} \operatorname{coth} \kappa_{0}(b-d), \quad$ for the $\mathrm{HE}_{m n}^{\text {slow }}$ mode

where the mode notation is chosen consistent with that used by Harrington [19]. An EH mode has a dominant magnitude of $E_{z}$, compared with that of $H_{z}$ and vice versa for an $\mathrm{HE}$ mode. In general, a fast wave has a phase velocity larger than the speed of light in vacuum, and a slow wave has a smaller phase velocity. Note that in the limit of large beam and waveguide cross sections $(a, b, t \rightarrow \infty ; d, \Delta, \delta \rightarrow 0)$, the EH mode dispersion equation (4) reduces to the familiar 1-D space-charge resonance equation:

$$
\omega=k_{z} v_{0} \pm \omega_{p} / \gamma_{0}^{3 / 2}
$$

In principle, we can obtain the complex wave number $k_{z}$ for an arbitrary beam-current density by solving the transcendental equations (4) and (5). To obtain an analytic solution of the growth rate near synchronism, we will consider a dilute beam in the following section.

\section{A Resonant Growth Rate for a Dilute Beam}

Conceptually, the complete beam-dielectric waveguide structure supports the coupled dielectric-lined guide waves and space-charge waves. The coupling becomes strong when phase velocities are close to each other. In this situation, the system is at synchronism and the coupled field is modified from the fields fro the uncoupled dielectric-lined guided wave and the beam space-charge wave. However, if the beam is dilute $\left(\chi_{b}<<1\right)$, the field modification due to the coupling becomes modest. Then the dispersion functions $D_{E}\left(\omega, k_{z}, \chi_{b}\right)$ and $D_{H}\left(\omega, k_{z}, \chi_{b}\right)$ can be expanded around $\chi_{b}=0$ and the resonance point $\left(\omega_{\text {res }}, k_{z}\right.$, res $)$ :

$$
\begin{aligned}
0= & D\left(\omega, k_{z}, \chi_{b}\right)=\left.D\left(\omega, k_{z} \chi_{b}\right)\right|_{\left(\omega_{\mathrm{res}}, k_{z, \mathrm{res}}, \chi_{b}=0\right)} \\
& +\left.\delta k_{z} \frac{\partial D\left(\chi_{b}=0\right)}{\partial k_{z}}\right|_{\left(\omega_{\mathrm{res}}, k_{z, \mathrm{res}}\right)} \\
& +\left.\chi_{b} \frac{\partial D}{\partial \chi_{b}}\right|_{\left(\omega_{\mathrm{res}}, k_{z, \mathrm{res}}, \chi_{b}=0\right)} .
\end{aligned}
$$

The resonance point $\left(\omega_{\text {res }}, k_{z \text {,res }}\right)$ simultaneously satisfies the uncoupled space-charge wave equation (10), and the uncoupled dielectric-lined dispersion equations, (7) and (9). Substituting these relationships into (11) leads to the solution for the perturbed wave number for the $\mathrm{EH}_{m n}$ mode:

$$
\begin{aligned}
\delta k_{z}^{(E)}= & {\left[\begin{array}{l}
-1 \\
\frac{1+\sqrt{3 i}}{2} \\
\frac{1+\sqrt{3 i}}{2}
\end{array}\right]\left[\frac{\omega_{p}^{2}}{2 \gamma_{0}^{3}} \frac{\kappa_{0} \kappa_{d}}{\omega v_{0}} \frac{k_{z}^{2}}{k_{x}^{2}+k_{z}^{2}}\right]^{1 / 3} } \\
& \cdot F_{E}^{1 / 3}\left(d, \delta, t, \Delta, \epsilon_{r}\right)
\end{aligned}
$$


with

$$
\begin{aligned}
F_{E}\left(d, \delta, t, \Delta, \epsilon_{r}\right)= & \left\{\frac{\kappa_{0} \epsilon_{r}}{1+\tanh \kappa_{0} \Delta \tanh \kappa_{0} \delta}\right\} \\
& \cdot\left\{\tanh \kappa_{0} \mathrm{t}\left[1-\tanh \kappa_{0} \Delta \tanh \kappa_{0} \delta+\tanh \kappa_{0}(\Delta+\delta)\left(\tanh \kappa_{0} \Delta-\tanh \kappa_{0} \delta\right)\right]\right. \\
& \left.-\frac{\kappa_{0} t}{\cosh ^{2} \kappa_{0} t}\left[1+\tanh \kappa_{0} \Delta \tanh \kappa_{0} \delta-\tanh \kappa_{0}(\Delta+\delta)\left(\tanh \kappa_{0} \Delta+\tanh \kappa_{0} \delta\right)\right]\right\} \\
& \cdot\left\{\kappa_{0}\left(\tan \kappa_{d} d+\kappa_{d} d \sec ^{2} \kappa_{d} d\right)+\kappa_{d} \epsilon_{r}\left[\tanh \kappa_{0}(\Delta+\delta)+\frac{\kappa_{0} \epsilon_{r}(\Delta+\delta)}{\cosh ^{2} \kappa_{0}(\Delta+\delta)}\right]\right\}^{-1} \\
& \stackrel{\tanh \kappa_{0} \Delta \rightarrow 1}{\longrightarrow} 2 \tanh \kappa_{0} t e^{-2 \kappa_{0} \delta} \frac{\epsilon_{r}}{\tan \kappa_{d} d+\kappa_{d} d \sec ^{2} \kappa_{d} d+\kappa_{d} \epsilon_{r} / \kappa_{0}}
\end{aligned}
$$

and for the $H E_{m n}$ mode,

$$
\begin{aligned}
\delta k_{z}^{(H)}= & {\left[\begin{array}{l}
-1 \\
\frac{1+\sqrt{3 i}}{2} \\
\frac{1+\sqrt{3 i}}{2}
\end{array}\right] a\left[\frac{\omega_{p}^{2}}{2 \gamma_{0}^{3}} \frac{\kappa_{0} \kappa_{d}}{\omega v_{0}} \frac{k_{x}^{2}}{k_{x}^{2}+k_{z}^{2}}\right]^{1 / 3} } \\
& \cdot F_{H}^{1 / 3}\left(d, \delta, t, \Delta, \epsilon_{r}\right)
\end{aligned}
$$

with

$$
\begin{aligned}
F_{H}\left(d, \delta, t, \Delta, \epsilon_{r}\right)= & \left\{\frac{\kappa_{0}\left[2+\left(\omega / \kappa_{0} c\right)^{2}\right]}{\operatorname{coth} \kappa_{0} \delta+\operatorname{coth} \kappa_{0} \Delta}\right\} \\
& \times\left\{\tanh \kappa_{0} t\left[\operatorname{coth} \kappa_{0} \delta-\operatorname{coth} \kappa_{0} \Delta+\operatorname{coth} \kappa_{0}(\Delta+\delta)\left(\operatorname{coth} \kappa_{o} \Delta \operatorname{coth} \kappa_{o} \delta-1\right)\right]\right. \\
& -\frac{\omega^{2}}{2\left(\kappa_{0} c\right)^{2}+\omega^{2}} \frac{\kappa_{0} t}{\cosh ^{2} \kappa_{0} t} \\
& \left.\cdot\left[\operatorname{coth} \kappa_{0} \delta+\operatorname{coth} \kappa_{0} \Delta-\operatorname{coth} \kappa_{0}(\Delta+\delta)\left(\operatorname{coth} \kappa_{0} \Delta \operatorname{coth} \kappa_{0} \delta+1\right)\right]\right\} \\
& \cdot\left\{\kappa_{0}\left(\kappa_{d} d \csc ^{2} \kappa_{d} d-\cot \kappa_{d} d\right)+\kappa_{d}\left[\operatorname{coth} \kappa_{0}(\Delta+\delta)-\frac{\kappa_{0}(\Delta+\delta)}{\left.\left.\sinh ^{2} \kappa_{0}(\Delta+\delta)\right]\right\}^{-1}}\right.\right. \\
& \stackrel{\tanh \kappa_{0} \Delta \rightarrow 1}{\longrightarrow} 2 \tanh \kappa_{0} t e^{-2 \kappa_{0} \delta} \frac{2+\left(\omega / \kappa_{0} c\right)^{2}}{\kappa_{d} d \csc ^{2} \kappa_{d} d-\cot \kappa_{d} d+\kappa_{d} / \kappa_{0}} \cdot
\end{aligned}
$$

Recall that a dilute beam $\chi_{b}<<1$ has been assumed for obtaining the above resonant growth rates. For consistency, the derived resonant grown rates in (12) and (13) have to be substituted into the expression of $\chi_{b}$ and rechecked. Hence, we obtain a constraint on the plasma frequency for the validity of the dilute beam model:

$$
\omega_{p}<<\omega_{\text {crit }}= \begin{cases}\frac{1}{2} \gamma_{0}^{3 / 2} \frac{\kappa_{0} \kappa_{d}}{k_{x}^{2}+k_{z}^{2}} \omega F_{E}, & \text { for the } E H_{m n} \text { mode } \\ \frac{1}{2} \gamma_{0}^{3 / 2} \frac{\kappa_{0} \kappa_{d}}{k_{x}^{2}+k_{z}^{2}} \frac{k_{x}^{2}}{k_{z}^{2}} \omega F_{H}, & \text { for the } H E_{m n} \text { mode }\end{cases}
$$



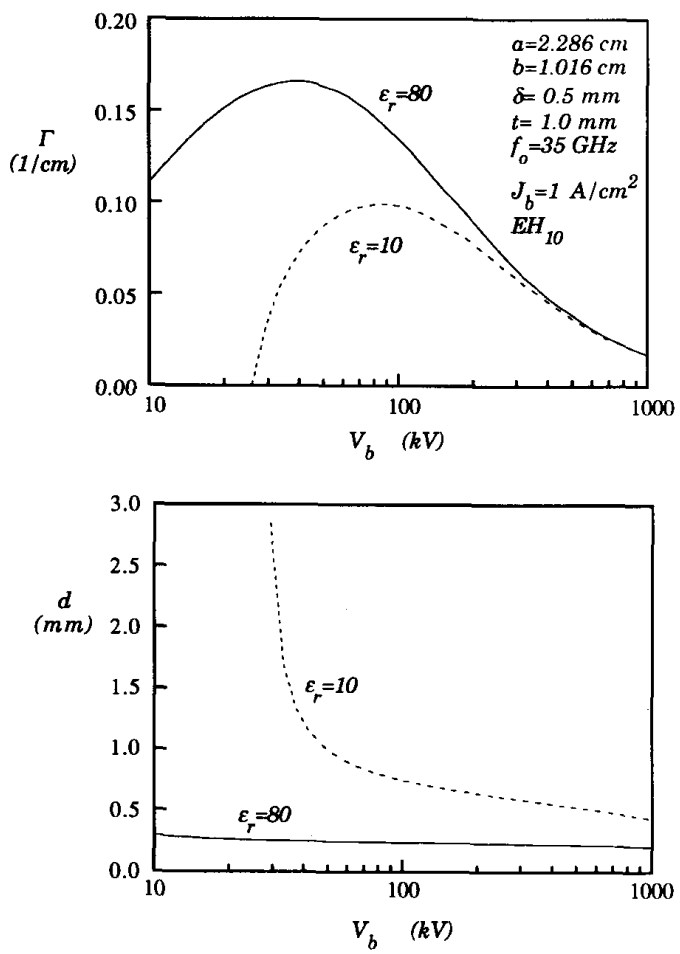

Fig. 2. Resonant growth rate and dielectric thickness versus the electron-beam voltage.

\section{Analytic Results of Resonant Growth Rate for a Dilute Beam}

The resonant growth and dielectric thickness versus beam voltage are shown in Fig. 2 for a rectangular waveguide with a size of 2.286 by $1.016 \mathrm{~cm}$ and a sheet beam with a current density of $1 \mathrm{~A} / \mathrm{cm}^{2}$. For a given beam voltage and operating frequency, the resonant dielectric thickness $d_{\text {res }}$ is determined from (7) or (9) and (10), which satisfy the Cerenkov synchronous condition. Inevitably, the beam-mode interaction will slightly shift the $d_{\text {res }}$ value from that calculated above. The growth rate is found to be $0.8-2 \mathrm{~dB} / \mathrm{cm}$. Note that the dielectric thickness is very sensitive to the variation of the beam voltage near threshold for Cerenkov amplification, and less sensitive far above the threshold. This implies that for low-beam voltages near the threshold operation regime, poor fabrication tolerances will lead to significantly reduced gains.

For higher power operation, the current density of a $40-\mathrm{kV}$ beam is increased to a value of $32 \mathrm{~A} / \mathrm{cm}^{2}$. One should note that for this range of current density, the condition $\chi_{b}<1$ is marginally satisfied (i.e., $\chi_{b} \sim 0.4$ ). The resonant growth rate calculated from (12) and (13), which is valid for a dilute beam $\left(\chi_{b}<<1\right)$, can only be considered as an optimistic prediction, and more accurate results must be solved directly from the dispersion equations (4) and (5). A resonant growth rate of $2 \mathrm{~dB} / \mathrm{cm}$ is obtained from (12) and an extraction efficiency of $3 \%$ is obtained from approximate beam-energy reduction based on conventional trapping arguments for the $\mathrm{EH}_{10}$ mode

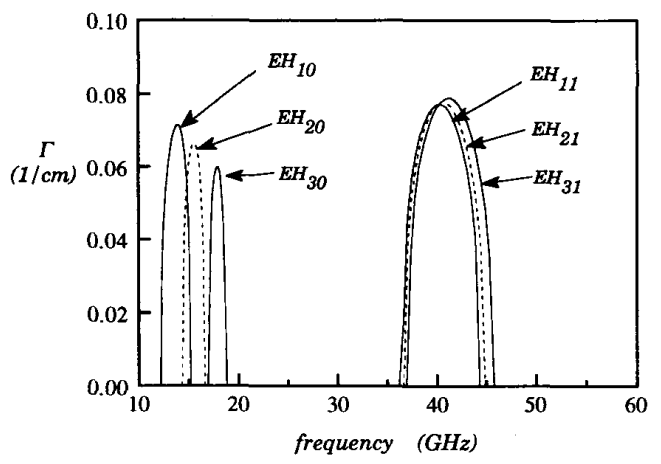

Fig. 3. Spectral competition of growing modes. System parameters are as follows: $a=2.286 \mathrm{~cm} ; b=1.016 \mathrm{~cm} ; \mathrm{d}=3 \mathrm{~mm} ; \delta=0.5 \mathrm{~mm}$; $t=1 \mathrm{~mm} ; \epsilon_{r}=10 ; V_{b}=40 \mathrm{kV}$; and $J_{b}=1 \mathrm{~A} / \mathrm{cm}^{2}$.

at $35 \mathrm{GHz}$ for $\epsilon_{r}=10$. The $E_{z}$-field decay length above the dielectric $\left(=2 \pi / \kappa_{0}\right)$ is about $5 \mathrm{~mm}$, which indicates that an electron beam must be placed within $5-\mathrm{mm}$ above the dielectric liner for a strong interaction. The saturation length is estimated to be $30 \mathrm{~cm}$ for a saturation power level of $8.7 \mathrm{~kW}$, estimated from the usual bounce length criterion obtained from ponderomotive theory [20].

Since the guide is over-sized, higher order modes are expected to compete with the fundamental $\mathrm{EH}_{10}$ mode. The competition in the frequency domain is plotted in Fig. 3, which shows that the $\mathrm{EH}_{20}$ and $\mathrm{EH}_{30}$ modes are the competing modes in the nearby frequency bands, whereas the $\mathrm{EH}_{m 1}(m=1,2, \cdots)$ modes compete in a higher frequency range. By careful mode coupler design, selective excitation of the $\mathrm{EH}_{10}^{\text {slow }}$ mode at the amplifier input can be ensured. This, along with nonlinear mode suppression effects [21], [22], should minimize mode competition. The mode converter design problem is discussed in the next section.

\section{HYBRID-MODE CONVERTER}

\section{A. Coupled Mode Equations}

The amplifier configuration we deal with is a constant crosssection guide filled with an inhomogeneous and anisotropic medium. The inhomogeneity comes from the dielectric liner and electron beam, which partially fill the waveguide. The anistropy is due to the electron motion in a strong magnetostatic field, where an electron beam is treated as an active medium with an anisotropic dielectric coefficient, as in (1). We generalize the hybrid-mode treatment of an isotropic dielectriclined guide by choosing eigenmodes of a waveguide filled with the inhomogeneous and anisotropic medium:

$$
\overline{\bar{\epsilon}}=\left[\begin{array}{ccc}
\epsilon_{x x} & 0 & 0 \\
0 & \epsilon_{y y} & 0 \\
0 & 0 & \epsilon_{z z}
\end{array}\right]
$$




$$
=\left\{\begin{array}{cll}
\epsilon_{0} \underline{\epsilon_{r}} \overline{\bar{I}}, & & 0<y<d(z) \\
\epsilon_{0} \overline{\bar{I}}, & & d(z)<y<b-\Delta-t \\
\epsilon_{0}\left[\begin{array}{ccc}
1 & 0 & 0 \\
0 & 1 & 0 \\
0 & 0 & 1+\chi_{b}
\end{array}\right], & b-\Delta-t<y<b-\Delta \\
\epsilon_{0} \overline{\bar{I}}, & & b-\Delta<y<b .
\end{array}\right.
$$

The susceptibility, $\chi_{b}$, can be a complex quantity due to the beam-mode interaction. The field is expanded in terms of local normal modes, which are eigenmodes of an artificial uniform guide with a cross section identical to that of the nonuniform guide at $z$. By use of the reciprocity theorem [23], the coupled-mode equations are obtained in terms of the wave forward and backward amplitudes as follows:

$$
\frac{d}{d z} \overline{\boldsymbol{A}}(z)=\overline{\bar{\gamma}}[(z)+\overline{\overline{\boldsymbol{K}}}(z)] \cdot \overline{\boldsymbol{A}}(z)
$$

where $\overline{\boldsymbol{A}}(0)=\overline{\boldsymbol{A}}_{0}$

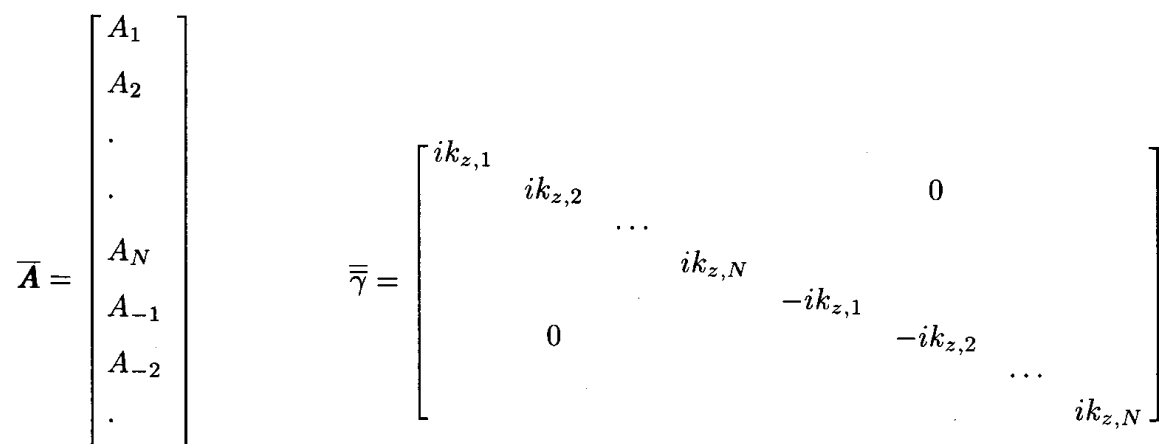

$$
\begin{aligned}
& A_{-N} \\
& \overline{\overline{\boldsymbol{K}}}=\left[\begin{array}{cccccccccc}
K_{1,1} & K_{1,2} & \cdot & \cdot & \cdot & K_{1,-1} & K_{1,-2} & \cdot & \cdot & \cdot \\
K_{2,1} & K_{2,2} & \cdot & \cdot & \cdot & K_{2,-1} & K_{2,-2} & \cdot & \cdot & \cdot \\
\cdot & \cdot & & & & \cdot & \cdot & & \\
\cdot & \cdot & & & & \cdot & \cdot & & \\
\cdot & \cdot & & & \cdot & \cdot & & \\
K_{-1,1} & K_{-1,2} & \cdot & \cdot & \cdot & K_{-1,-1} & K_{-1,-2} & \cdot & \cdot & \cdot \\
K_{-2,1} & K_{-2,2} & \cdot & \cdot & \cdot & K_{-2,-1} & K_{-2,-2} & \cdot & \cdot & \cdot \\
\cdot & \cdot & & & \cdot & \cdot & & & \\
\cdot & \cdot & & & \cdot & \cdot & & & \\
\cdot & \cdot & & & & \cdot & \cdot & &
\end{array}\right] \\
& K_{m n}=-\frac{\operatorname{sgn}(m)}{2 N_{m}} \iint_{A_{g}}\left(\hat{\boldsymbol{e}}_{m} \times \frac{\partial \hat{\boldsymbol{h}}_{n}}{\partial z}+\frac{\partial \hat{\boldsymbol{e}}_{n}}{\partial z} \times \hat{\boldsymbol{h}}_{m}\right) \cdot \hat{\boldsymbol{z}} d a .
\end{aligned}
$$

In the above expressions, the positive index has been adopted for the mode propagating in the positive $z$ direction, and the negative index is used for negative $z$ propagation. The set of coupled mode equations derived above includes forward and backward mode-mode coupling, multiple coupling, and the beam-mode interaction. Since the integrand in (17) involves taking the $z$-derivative of eigenfields, the coupling coefficient $K_{m n}$ is very inconvenient to manipulate. Since the beam-mode interaction mainly occurs in the uniform Cerenkov interaction region, we ignore the beam-mode coupling effect in the converter region; i.e., $\overline{\bar{\epsilon}}$ is considered lossless. Consequently, the form of $K_{m n}$ is simplified further as follows: 


$$
\begin{aligned}
K_{m n}= & -\frac{1}{2} \iint_{A_{g}}\left(\hat{\boldsymbol{e}}_{m}^{*} \times \frac{\partial \hat{\boldsymbol{h}}_{n}}{\partial z}+\frac{\partial \hat{\boldsymbol{e}}_{n}}{\partial z} \times \hat{\boldsymbol{h}}_{m}^{*}\right) \cdot \hat{\boldsymbol{n}} d a \\
& = \begin{cases}-\frac{s g n(m)}{2} \frac{\omega \epsilon_{0}}{k_{z, n}-k_{z, m}} \iint_{A_{g}} \frac{\partial \epsilon_{n}}{\partial z} \hat{\boldsymbol{e}}_{m}^{*} \cdot \hat{\boldsymbol{e}}_{n} d a, & |m| \neq|n| \\
\frac{s g n(m)}{4} \frac{\omega \epsilon_{0}}{k_{z, m}} \iint_{A_{g}} \frac{\partial \epsilon_{r}}{\partial z}\left|\hat{\boldsymbol{e}}_{m}\right|^{2} d a, & m=-n \\
-\frac{\operatorname{sgn}(m)}{2} \iint_{A_{g}}\left(\hat{\boldsymbol{e}}_{m}^{*} \times \frac{\partial \hat{\boldsymbol{h}}_{m}}{\partial z}+\frac{\partial \hat{\boldsymbol{e}}_{m}}{\partial z} \times \hat{\boldsymbol{h}}_{m}^{*}\right) \cdot \hat{\boldsymbol{z}} d a, & m=n .\end{cases}
\end{aligned}
$$

\section{B. Analytic Solutions Along the Mode Converter}

We shall obtain an analytic form of solution for a gradual converter, where numerous waveguide modes can propagate simultaneously. The differential matrix equation (16) is very difficult to solve analytically unless the matrix $\overline{\bar{M}}(z)=$ $\overline{\bar{\gamma}}(z)+\overline{\overline{\boldsymbol{K}}}(z)$ is a triangular matrix. To resolve this difficulty, we will adopt the approach of [24], which was developed for a smooth-wall mode converter. The differential matrix equation is first transformed by a similarity transformation $\overline{\boldsymbol{A}}(z)=$ $\overline{\overline{\boldsymbol{P}}}(z) \cdot \overline{\boldsymbol{B}}(z)$. The choice of $\overline{\overline{\boldsymbol{P}}}(z)$ is based on the observation that the problem essentially describes a physical problem of coupled harmonic oscillators. The transfer is equivalent to the procedure of looking for the uncoupled normal modes of the system, which are the eigenvectors of $\overline{\bar{M}}$. Therefore, the transform matrix $\overline{\bar{P}}$, composed of these eignevectors, is a diagonalizing matrix, which diagonalizes the matrix $\overline{\bar{M}}$ to the matrix $\overline{\bar{q}}$. However, since the coupling coefficients are spatially varying, $\overline{\bar{P}}(z)$ becomes a quasi-diagonalizing matrix for $\overline{\overline{\boldsymbol{M}}}(z)$. This transformation yields a diagonalized matrix $\overline{\overline{\boldsymbol{q}}}$, with an additional off-diagonal matrix $\overline{\bar{e}}(z)$. For a gentle taper rate converter, $\overline{\bar{e}}(z)$ is a small variable, and the transformed differential matrix equations are solved in orders of $\overline{\bar{e}}(z)$ :

$$
\overline{\boldsymbol{A}}(z)=\overline{\overline{\boldsymbol{P}}}(z) \cdot \overline{\overline{\boldsymbol{G}}}(z) \cdot \overline{\boldsymbol{A}}_{0}
$$

where

$$
\begin{aligned}
\overline{\overline{\boldsymbol{G}}}(z) & =\overline{\overline{\boldsymbol{G}}}^{(0)}(z)+\overline{\overline{\boldsymbol{G}}}^{(1)}(z)+\overline{\overline{\boldsymbol{G}}}^{(2)}(z)+\cdots \\
\overline{\overline{\boldsymbol{G}}}^{(0)}(z) & =e^{\int_{0}^{z} \overline{\overline{\boldsymbol{q}}}\left(z^{\prime}\right) d z^{\prime}}
\end{aligned}
$$

$$
\begin{gathered}
\overline{\overline{\boldsymbol{G}}}^{(1)}(z)=-e^{\int_{0}^{z} \overline{\overline{\boldsymbol{q}}}\left(z^{\prime}\right) d z^{\prime}} \cdot \int_{0}^{z} d z^{\prime} \exp \left\{-\int_{0}^{z^{\prime}} \overline{\overline{\boldsymbol{q}}}\left(z^{\prime \prime}\right) d z^{\prime \prime}\right\} \\
\cdot \overline{\overline{\boldsymbol{e}}}\left(z^{\prime}\right) \exp \left\{\int_{0}^{z^{\prime}} \overline{\overline{\boldsymbol{q}}}\left(z^{\prime \prime}\right) d z^{\prime \prime}\right\}
\end{gathered}
$$

$$
\begin{aligned}
\overline{\overline{\boldsymbol{G}}}^{(2)}(z)= & e^{\int_{0}^{z} \overline{\overline{\boldsymbol{q}}}\left(z^{\prime}\right) d z^{\prime}} \cdot \int_{0}^{z} d z^{\prime}\left[\exp \left\{-\int_{0}^{z^{\prime}} \overline{\overline{\boldsymbol{q}}}\left(z^{\prime \prime}\right) d z^{\prime \prime}\right\}\right. \\
& \left.\cdot \overline{\overline{\boldsymbol{e}}}\left(z^{\prime}\right) \cdot \exp \left\{\int_{0}^{z^{\prime}} \overline{\overline{\boldsymbol{q}}}\left(z^{\prime \prime}\right) d z^{\prime \prime}\right\}\right] \\
& \cdot\left[\int_{0}^{z^{\prime}} d z^{\prime \prime} \exp \left\{-\int_{0}^{z^{\prime \prime}} \overline{\overline{\boldsymbol{q}}}\left(z^{\prime \prime \prime}\right) d z^{\prime \prime \prime}\right\} \cdot \overline{\overline{\boldsymbol{e}}}\left(z^{\prime \prime}\right)\right. \\
& \left.\cdot \exp \left\{\int_{0}^{z^{\prime \prime}} \overline{\overline{\boldsymbol{q}}}\left(z^{\prime \prime \prime}\right) d z^{\prime \prime \prime}\right\}\right]
\end{aligned}
$$

In principle, this formulation can be used for an arbitrary number of modes, provided that the eignevectors and eigenvalues of $\overline{\bar{M}}(z)$ are obtainable. It is relatively easy for the two-dimensional $\overline{\bar{M}}(z)$, but it becomes cumbersome when higher order dimensions are considered. To obtain an explicit analytic solution, we focus our efforts on the two-mode forward coupling.

The dielectric-lines converter under consideration is assumed to only be tapered in the $y$ dimension, so that only those modes with the same field distribution in the $x$ dimension are coupled. This leads to the selection rule that coupling exists among those modes with the same $m$ index. Moreover, from (19), strong coupling occurs for those modes propagating in the same direction and with close phase velocities. For further simplification, only the direct coupling between any two modes is considered; i.e., recoupling through the third mode is neglected. We can then restrict our attention to only two modes at a time. The coupled mode equation is now recast as

$$
\frac{d}{d z}\left[\begin{array}{l}
A_{1} \\
A_{2}
\end{array}\right]=\left[\begin{array}{cc}
i k_{z, 1} & C \\
-C & i k_{z, 2}
\end{array}\right]\left[\begin{array}{l}
A_{1} \\
A_{2}
\end{array}\right]
$$

where the coupling coefficient $C(z)$ is calculated from (19) as follows.

$$
\begin{aligned}
& \text { 1) } E H_{m n}^{\text {fast }} \rightarrow E H_{m \ell}^{\text {fast }}: \\
& C=-\frac{a}{4 F_{m}} \frac{\omega \epsilon_{0}\left(\epsilon_{r}-1\right)}{k_{z, m \ell}-k_{z, m n}} d^{\prime}(z)(-1)^{n+\ell} N_{m n} N_{m \ell} \\
& \cdot\left|\cos \kappa_{0, m n}(b-d)\right|\left|\cos \kappa_{0, m \ell}(b-d)\right|
\end{aligned}
$$




$$
\begin{array}{r}
{\left[\sin \kappa_{d, m n} d \sin \kappa_{d, m \ell} d \frac{\kappa_{d, m n} \kappa_{d, m \ell}\left(k_{x}^{2}+k_{z, m n} k_{z, m \ell}\right)}{\left(k_{x}^{2}+k_{z, m n}^{2}\right)\left(k_{x}^{2}+k_{z, m \ell}^{2}\right)}\right.} \\
\left.+\cos \kappa_{d, m n} d \cos \kappa_{d, m \ell} d\right] .
\end{array}
$$$$
\text { 2) } E H_{m n}^{f a s t} \rightarrow E H_{m \ell}^{f a s t} \text { : }
$$$$
C=\frac{a}{4 F_{m}} \omega^{2} \mu_{0} \epsilon_{0}\left(\epsilon_{r}-1\right) d^{\prime}(z)(-1)^{n+\ell+1} N_{m n} N_{m \ell}
$$$$
\text { - }\left|\cos \kappa_{0, m n}(b-d)\right|\left|\sin \kappa_{0, m \ell}(b-d)\right|
$$$$
\cdot \sin \kappa_{d, m n} d \sin \kappa_{d, m \ell} d \frac{\kappa_{d, m n} k_{x}}{\left(k_{x}^{2}+k_{z, m n}^{2}\right)\left(k_{x}^{2}+k_{z, m \ell}^{2}\right)} .
$$$$
\text { 3) } E H_{m n}^{\text {slow }} \rightarrow E H_{m \ell}^{\text {slow }} \text { : }
$$$$
C=-\frac{a}{4 F_{m}} \frac{\omega \epsilon_{0}\left(\epsilon_{r}-1\right)}{k_{z, m \ell}-k_{z, m n}} d^{\prime}(z) N_{m n} N_{m \ell}
$$$$
\cdot \cosh \kappa_{0, m n}(b-d) \cosh \kappa_{0, m \ell}(b-d)
$$$$
\begin{array}{r}
{\left[\sin \kappa_{d, m n} d \sin \kappa_{d, m \ell} d \frac{\kappa_{d, m n} \kappa_{d, m \ell}\left(k_{x}^{2}+k_{z, m n} k_{z, m \ell}\right)}{\left(k_{x}^{2}+k_{z, m n}^{2}\right)\left(k_{x}^{2}+k_{z, m \ell}^{2}\right)}\right.} \\
\left.+\cos \kappa_{d, m n} d \cos \kappa_{d, m \ell} d\right]
\end{array}
$$

4) $E H_{m n}^{\text {slow }} \rightarrow H E_{m \ell}^{\text {slow }}$ :

$C=\frac{a}{4 F_{m}} \omega^{2} \mu_{0} \epsilon_{0}\left(\epsilon_{r}-1\right) d^{\prime}(z) N_{m n} N_{m \ell}$

- $\cosh \kappa_{0, m n}(b-d) \sinh \kappa_{0, m \ell}(b-d)$

$$
\cdot \sin \kappa_{d, m n} d \sin \kappa_{d, m \ell} d \frac{\kappa_{d, m n} k_{x}}{\left(k_{x}^{2}+k_{z, m n}^{2}\right)\left(k_{x}^{2}+k_{z, m \ell}^{2}\right)} .
$$

5) $E H_{m n}^{\text {slow }} \rightarrow E H_{m \ell}^{\text {slow }}$ :

$$
\begin{aligned}
& C=-\frac{a}{4 F_{m}} \frac{\omega \epsilon_{0}\left(\epsilon_{r}-1\right)}{k_{z, m \ell}-k_{z, m n}} d^{\prime}(z)(-1)^{\ell} N_{m n} N_{m \ell} \\
& \cdot \cosh \kappa_{0, m n}(b-d)\left|\cos \kappa_{0, m \ell}(b-d)\right| \\
& \cdot\left[\begin{array}{c}
\sin \kappa_{d, m n} d \sin \kappa_{d, m \ell} d \frac{\kappa_{d, m n} \kappa_{d, m \ell}\left(k_{x}^{2}+k_{z, m n} k_{z, m \ell}\right)}{\left(k_{x}^{2}+k_{z, m n}^{2}\right)\left(k_{x}^{2}+k_{z, m \ell}^{2}\right)} \\
\left.+\cos \kappa_{d, m n} d \cos \kappa_{d, m \ell} d\right] .
\end{array}\right.
\end{aligned}
$$

$$
\begin{aligned}
& \text { 6) } \begin{aligned}
E & H_{m n}^{s l o w} \rightarrow H E_{m \ell}^{\text {fast }}: \\
C= & \frac{a}{4 F_{m}} \omega^{2} \mu_{0} \epsilon_{0}\left(\epsilon_{r}-1\right) d^{\prime}(z)(-1)^{\ell+1} N_{m n} N_{m \ell} \\
& \cdot \cosh \kappa_{0, m n}(b-d) \mid \sin \kappa_{0, m \ell}(b-d) \\
& \cdot \sin \kappa_{d, m n} d \sin \kappa_{d, m \ell} d \frac{\kappa_{d, m n} k_{x}}{\left(k_{x}^{2}+k_{z, m n}^{2}\right)\left(k_{x}^{2}+k_{z, m \ell}^{2}\right)}
\end{aligned}
\end{aligned}
$$

Eventually, the evolution of the coupled modes along the converter is obtained:

$$
\begin{aligned}
A_{1}(z)= & A_{1}(0)\left[\cos \theta(z) e^{i \rho_{d}}+i \sin \theta(z)\right. \\
& \left.\cdot e^{i \rho_{s}}[W(z)-i T(z)]\right] \\
& +A_{2}(0)\left[i \sin \theta(z) e^{i \rho_{s}}-\cos \theta(z)\right.
\end{aligned}
$$

$$
\begin{gathered}
\left.\cdot e^{i \rho_{d}}[W(z)-i T(z)]^{*}\right] \\
A_{2}(z)=-A_{1}(0)\left[i \sin \theta(z) e^{i \rho_{d}}+\cos \theta(z)\right. \\
\left.\cdot e^{i \rho_{s}}[W(z)-i T(z)]\right] \\
+A_{2}(0)\left[\cos \theta(z) e^{i \rho_{s}}+i \sin \theta(z)\right. \\
\left.\cdot e^{i \rho_{d}}[W(z)-i T(z)]^{*}\right]
\end{gathered}
$$

where

$$
\begin{aligned}
W(z) & =\int_{0}^{\rho} 2 \theta e^{-i 2 \rho^{\prime}} d \rho^{\prime} \\
T(z) & =\theta(z) e^{-2 i \rho(z)}-\theta(0) e^{-2 i \rho(0)} \\
\beta(z) & =\frac{1}{2}\left(k_{z, 1}(z)+k_{z, 2}(z)\right) \\
\Delta \beta(z) & =\frac{1}{2}\left(k_{z, 1}(z)-k_{z, 2}(z)\right) \\
\tan 2 \theta & =\frac{C(z)}{\Delta \beta(z)}, \quad \Gamma(z)=\sqrt{\Delta \beta(z)^{2}+C(z)^{2}} \\
\rho(z) & =\int_{0}^{z} \Gamma\left(z^{\prime}\right) d z^{\prime}, \quad \rho_{d}(z)=\int_{0}^{z}\left[\beta\left(z^{\prime}\right)-\Gamma\left(z^{\prime}\right)\right] d z^{\prime} \\
\rho_{s}(z)= & \int_{0}^{z}\left[\beta\left(z^{\prime}\right)+\Gamma\left(z^{\prime}\right)\right] d z^{\prime} .
\end{aligned}
$$

Note that $\rho_{\boldsymbol{d}}$ and $\rho_{s}$ are the phase functions of the quasi-normal modes of the system, and $\rho$ can be interpreted as a phase accumulation due to the axial variation of coupling coefficient.

Before elaborating on the mode evolution along the converter, we examine the mode conversion path as the dielectric thickness varies. It is demonstrated in Fig. 4 that the empty $\mathrm{TE}_{m 0}$ mode is converted into the partially filled $\mathrm{EH}_{m 0}$ mode, and then evolves into the fully filled $\mathrm{TE}_{m 0}$ mode. Likewise, the empty $\mathrm{TE}_{0 n}$ mode converts to the $\mathrm{HE}_{0 n}$ mode, and back to the fully filled $\mathrm{TE}_{0 n}$ mode. In addition, the $\mathrm{HE}_{11}$ and $\mathrm{EH}_{11}$ modes merge into the degenerate empty $\mathrm{TE}_{11}$ and $\mathrm{TM}_{11}$ modes. Except for the $\mathrm{EH}_{m 0}$ modes, each mode changes its characteristics from a fast wave to a slow wave, depending on the dielectric parameters and frequency.

\section{Linear Taper for a $T E_{10}^{\text {last }}$-to-E $H_{10}^{\text {slow }}$ Mode Converter}

Consider a simple linear taper with $z_{\text {tap }}=10 \mathrm{~cm}$ in a waveguide with the size of $a \times b=2.286 \times 1.016 \mathrm{~cm}^{2}$ (WR90), a dielectric constant of $\epsilon_{r}=10$, and a thickness varying from zero to $d_{\text {res }}=1.2 \mathrm{~mm}$. The value of $d_{\text {res }}$ is determined from (7) and (10)-i.e., the Cerenkov synchronous condition at $35 \mathrm{GHz}$ for the $\mathrm{EH}_{10}$ mode and a beam voltage of $40 \mathrm{kV}$.

The mode conversion functions $2 \theta\left(\equiv \tan ^{-1} 2 C / \Delta \beta\right)$ for $\mathrm{EH}_{10}$-to- $\mathrm{EH}_{11}, \mathrm{EH}_{10}$-to- $\mathrm{EH}_{12}, \mathrm{EH}_{10}$-to-HE $\mathrm{HE}_{11}$, and $\mathrm{EH}_{10}$-to$\mathrm{EH}_{11}$ (backward) modes are plotted in Fig. 5. It indicates that the accumulation of mode conversion from $\mathrm{EH}_{10}$-to- $\mathrm{HE}_{11}$ forward coupling along the taper is dominant. In addition, each curve has a peak value at a particular axial location, determined by the wave number difference and the field scalar-product contributions to the coupling strength. Although the mode 


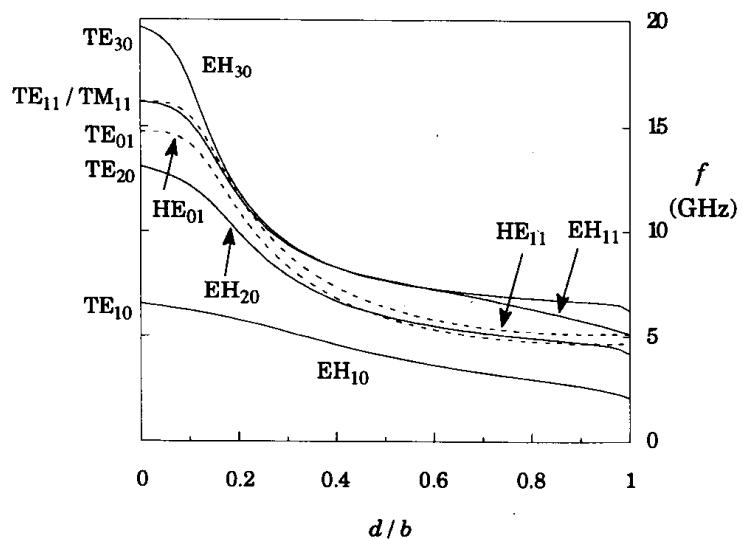

Fig. 4. Cut-off frequencies for various normal modes versus the dielectric filling ratio $d / b$ ( $d$ : the dielectric thickness. $b$ : the waveguide height).

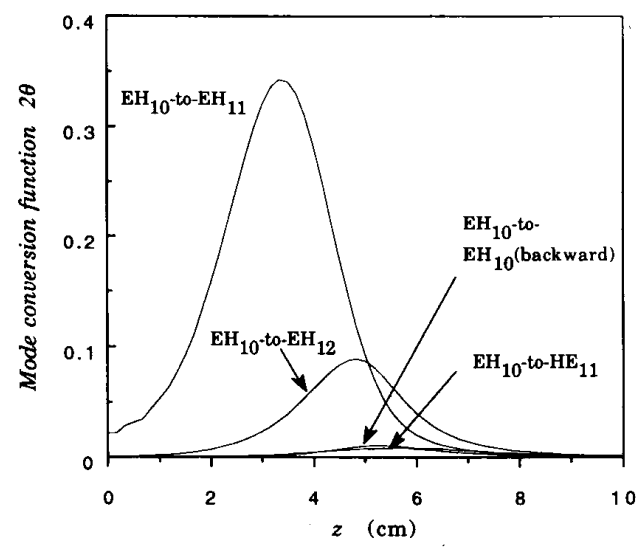

Fig. 5. Mode conversion functions of $\mathrm{EH}_{10}-\mathrm{to}-\mathrm{EH}_{11}, \mathrm{EH}_{10}$-to- $\mathrm{EH}_{12}$, $\mathrm{EH}_{10}$-to- $\mathrm{HE}_{11}$, and $\mathrm{EH}_{10}$-to- $\mathrm{EH}_{11}$ (backward) for a linear taper. System parameters: $a=2.286 \mathrm{~cm} ; b+1.016 \mathrm{~cm} ; \epsilon_{r}=10 ; d_{\text {res }}=1.2 \mathrm{~mm}$; and $f_{0}=35 \mathrm{GHz}$.

separation (and phase velocity difference) is small initially, the field modification from a vacuum wave-guide field is less sensitive to variations in the dielectric thickness in this dielectric thickness range. At the other end of the taper, the mode separation is larger due to the comparatively thicker liner which consequently weakens the coupling strength. Hence, the largest mode coupling occurs in the middle region of a linear taper, as seen in Fig. 5 .

The evolution of $\mathrm{EH}_{11}, \mathrm{EH}_{12}$, and $\mathrm{HE}_{11}$ modes for an incident $\mathrm{TE}_{10}$ mode along the converter is shown in Fig. 6 . The dependence of the coupling coefficient, $c(z)$, on the spatial derivative of the dielectric thickness $d^{\prime}(z)$ [cf. expressions below (23)] results in a small amount of mode conversion at the beginning of the taper $(z=0)$ due to a discontinuity in the derivative, $d^{\prime}(z)$. We analytically calculate the effect of this discontinuity and use the result to set initial conditions at $z=0$ for the various modes displayed in Fig. 6. The $\mathrm{TE}_{10}$ mode primarily converts into the $\mathrm{EH}_{10}$ mode, together with coupling to higher order modes, as soon as the coupling mechanism

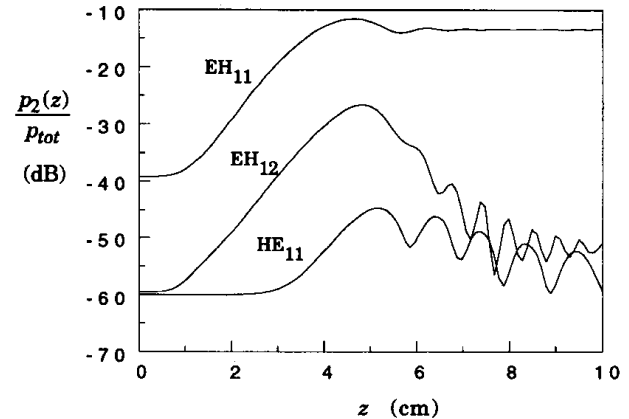

Fig. 6. Power evolution of $\mathrm{EH}_{11}, \mathrm{EH}_{12}$, and $\mathrm{HE}_{11}$ modes along the liner profile converter at $35 \mathrm{GHz}$ from the two-mode calculation.

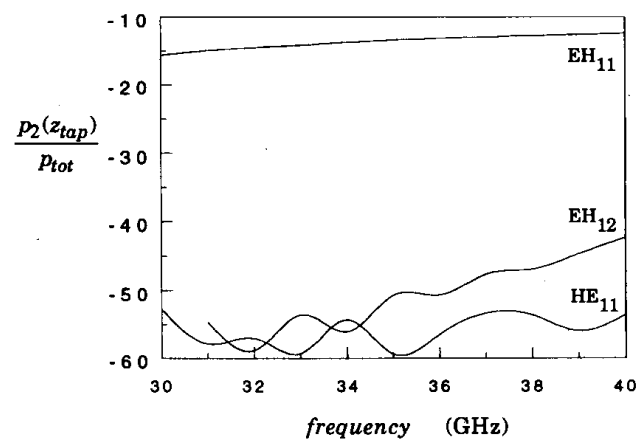

Fig. 7. Spectral evolution of resultant coupled $\mathrm{EH}_{11}, \mathrm{EH}_{12}$, and $\mathrm{HE}_{11}$ modes for a linear-profile converter.

starts. The power is coupled back and forth between modes, which corresponds to the oscillating part of the curves in Fig. 6. Among the undesired coupled modes, the $\mathrm{EH}_{11}$ power is at least $20 \mathrm{~dB}$ larger than either the $\mathrm{EH}_{12}$ or $\mathrm{HE}_{11}$ modes. The resultant $\mathrm{EH}_{11}$ mode at the end of the taper has $4.6 \%$ (or $-13.4 \mathrm{~dB}$ ) of the total input power. Regarding frequency response, Fig. 7 illustrates that the $\mathrm{EH}_{11}$ mode is the dominant coupled mode over the 30 - to $40 \mathrm{GHz}$ range.

\section{An Improved Taper Profile}

To improve the performance of a mode converter, several optimization schemes have been proposed for the smooth-wall and perturbed-wall mode converters. References [24]-[26] optimize the profile in $(2 \theta, \rho)$ space and then transform back into physical $(a, z)$ space, where $2 \theta$ and $\rho$ are defined in the same way as in (26), and $a$ is the radius of the smooth-wall cylindrical guide. In contrast, Lawson [27] was able to design a generalized-raised-cosine taper profile directly in $(a, z)$ space which shows better performance than other profiles over a specified frequency range: Schill and Seshadri [28], and Luo et al. [29], [30] give optimization criteria for maximizing the conversion efficiency by perturbing the guide radius. The essential difference in the configuration under consideration here is that a high-dielectric constant liner, necessary for lowvoltage Cerenkov interaction, is to be tapered up from zero to several millimeters. To up-taper this thin dielectric liner with an optimized general profile is mechanically impractical. A 


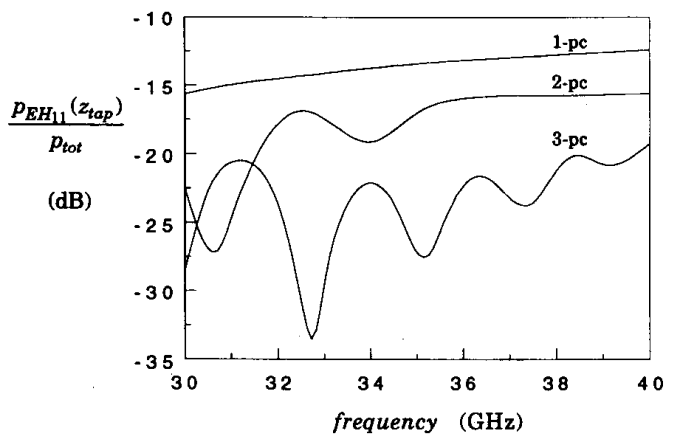

Fig. 8. Comparison of the spectral response of coupled $\mathrm{EH}_{11}$ power for various piece-wise linear tapers.

linear of piece-wise linear profile is preferable. Hence, in the following we will improve the linear taper using a piece-wise profile.

Examining the form of the coupling coefficient in (23), we can decompose it as the product of taper rate $\frac{d}{d z}(d(z))$ and the other term, denoted as $\bar{C}$, which is explicitly independent of the taper rate. An improvement criterion is described as follows. The taper rate is chosen small whenever $\bar{C}$ becomes large under the constraint that the total taper length and final dielectric thickness are fixed.

Two-piece and a three-piece linear tapers are calculated, respectively. The $\mathrm{EH}_{11}$ power is dramatically reduced to $2.0 \%$ $(-17 \mathrm{~dB})$ and $0.2 \%(-27 \mathrm{~dB})$ at $35 \mathrm{GHz}$ for the 2-piece and 3-piece linear tapers as shown in Fig. 8. The respective taper profiles are depicted in Fig. 9. Over the 30- to $40-\mathrm{GHz}$ range, the 3-piece taper has coupled $\mathrm{EH}_{11}$ power at least 8and 5-dB lower than that of the 1-piece and 1-piece tapers, respectively. We then examine other coupled modes for the improved 3-piece taper profile. Fig. 10 shows that the $\mathrm{EH}_{12}$ and $\mathrm{HE}_{11}$ power levels are much less than the $\mathrm{EH}_{11}$ mode for $35 \mathrm{GHz}$ and beyond, the $\mathrm{EH}_{12}$ mode becomes significant below $30 \mathrm{GHz}$. This is due to the fact that the $\mathrm{EH}_{12}$ mode is near cut off at $30 \mathrm{GHz}$. Note that for modes near cut off, backward wave coupling becomes important and is neglected in our analysis of two-mode forward coupling; therefore the calculation is no longer valid. Naturally, we may increase the number of taper segments for further improvement, but the complexity of fabrication would offset the gain.

\section{A CERENKOV AMPLIFIER SYSTEM}

For a complete Cerenkov amplifier, a sheet electron beam is injected through a slow-wave guiding structure, which consists of an input mode converter, an interaction region, and an output mode converter, as shown in Fig. 11. Regarding the sheet-beam generation and transport, a concern is that beam rotation, deformation, breakup, and diocotron instability are associated with the solenoidal magnetic guiding field. Substantial experimental and theoretical work for conventional microwave tubes has been conducted since the 1950's [7]-[10], [31], [35]. A worst-case estimation for the diocotron instability growth

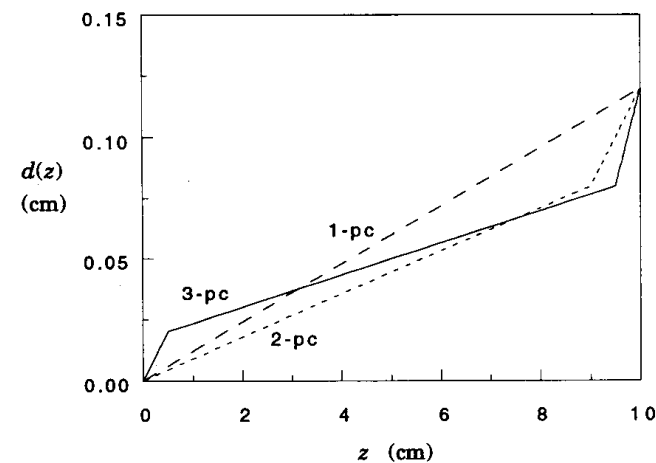

Fig. 9. Taper profiles of various piece-wise linear tapers.

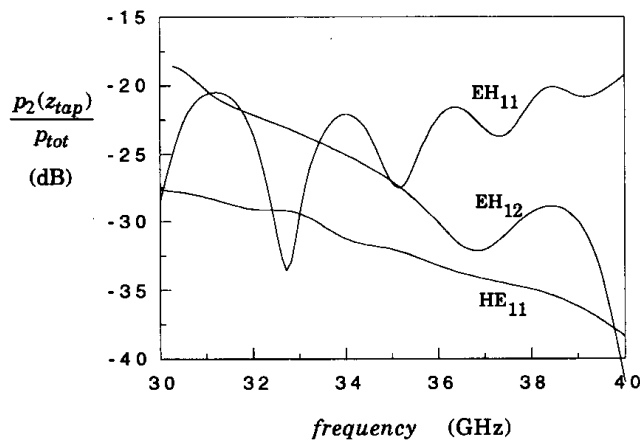

Fig. 10. Spectral evolution of resultant coupled $\mathrm{EH}_{11}, \mathrm{EH}_{12}$, and $\mathrm{HE}_{11}$ modes for a three-piece linear taper.

length can be obtained from [5]-[7], recast as follows:

$$
L_{\mathrm{e}}>v_{z} \frac{\omega_{c}}{\omega_{p}^{2}} \approx 800 \beta_{z}^{2} \frac{B_{z}(\mathrm{kG})}{J_{b}\left(\mathrm{~A} / \mathrm{cm}^{2}\right)} \quad(\mathrm{cm}) .
$$

For example, the diocotron growth length is $30 \mathrm{~cm}$ for a 40 $\mathrm{kV}, 10-\mathrm{A} / \mathrm{cm}^{2}$ beam of $1-\mathrm{mm}$ thickness confined by a $4-\mathrm{kG}$ magnetic field, which is marginally sufficient for millimeterwave amplifiers. Higher magnetic fields tend to suppress the instability. Regarding beam generation, Booske et al. [1], [2] have developed a sheet beam in the high-voltage regime with a wiggler confinement scheme, and Ferendeci [3] is designing a sheet beam with a current of $10 \mathrm{~A}$ and size of 2 by $20 \mathrm{~mm}$ for planar gyrotron applications. Furthermore, the sheet-beam generation and stable confinement by a sufficiently strong magnetic field has been demonstrated by Read et al. [4] for use in a gyrotron.

For the mode evolution in the input mode converter, the analytic results from two-mode coupling theory, obtained in Section III, can be applied to the region in the mode converter, but the theory is not self-consistent very close to the Cerenkov interaction region. This is because the beamwave Cerenkov interaction becomes strong when the beam and slow waves approach the interaction region. The mode converter is designed to convert the launched $\mathrm{TE}_{10}$ mode to the desired $\mathrm{EH}_{10}$ mode, but the unwanted $\mathrm{EH}_{11}, \mathrm{EH}_{12}$, and $\mathrm{HE}_{11}$ modes are excited also. 


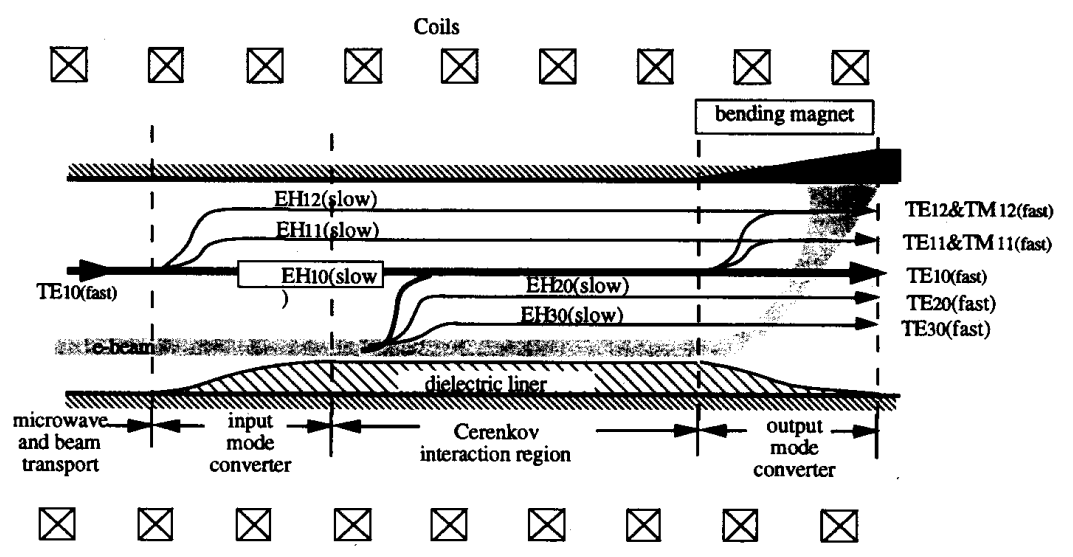

Fig. 11. Schematic sketch of a dielectric Cerenkov amplifier system.

After entering the interaction region, the beam-wave evolution can be described by the results from Section II. Typically, the desired $\mathrm{EH}_{10}$ mode is amplified by the beam with a growth rate of $0.8-2 \mathrm{~dB} / \mathrm{cm}$. The strongly competing modes include the $\mathrm{EH}_{20}$ and $\mathrm{EH}_{30}$ modes, in contrast to the $\mathrm{EH}_{11}$ mode in the mode converter. The spatial range of validity of this smallsignal analysis is determined by the inverse spatial growth length for the beam diocotron instability and the distance for nonlinear saturation of the beam-wave interaction, depending on which is shortest.

At the end of the Cerenkov interaction length, an output mode converter is used to couple the show-wave power while the electron beam is dumped in the collector. The output mode converter can be treated as the reciprocal of the input mode converter. This is possible because the beam-wave interaction is very weak in the output converter where the beam is deflected.

\section{CONCLUSIONS}

We have examined a dielectric Cerenkov maser amplifier, including space-charge collective effects in a rectangular cross-section structure. This configuration supports hybrid waveguide modes instead of the conventional TE and TM modes. A streaming electron beam confined by a strong magnetic field was treated as a cold fluid by assuming that electron-cyclotron effects are negligible. The dispersion relation for the sheet beam and hybrid mode linear interaction was obtained by solving an equivalent multilayer problem. Instead of numerically solving the transcendental dispersion equation, a growth rate near synchronism was analytically calculated in the low-current density limit. The saturation efficiency and saturation length, $L_{\text {sat }}$ were also estimated. The validity of the resonant growth rate calculation requires $\chi_{b}<<1$ and $z<<L_{\text {sat }}$.

We found that a low-voltage and moderate current density beam $\left(40 \mathrm{kV}\right.$ and $\left.1 \mathrm{~A} / \mathrm{cm}^{2}\right)$ can amplify the $\mathrm{EH}_{10}$ mode of a dielectric-lined rectangular guide $\left(\epsilon_{r}=10-80\right)$, with a $0.8-2 \mathrm{~dB} / \mathrm{cm}$ growth rate in the $35-\mathrm{GHz}$ range. The $\mathrm{EH}_{20}$ and $\mathrm{EH}_{30}$ modes also yield significant growth rates in slightly different frequency bands. Nonlinear mode suppression and slight modification of the guide dimensions should provide adequate mode selectivity.

We have derived a general set of coupled mode equations valid for a dielectric-lined mode converter of rectangular geometry, which can be used as an input or output coupler for the Cerenkov interaction section. The forward and backward coupling, multicoupling, and beam-mode interaction effects were included in the derived coupled-mode equations.

Since the beam-mode interaction mainly occurs in the uniform Cerenkov interaction region, the coupled-mode equations were further simplified by neglecting the beam-mode interaction effects in the tapered coupler. With this assumption, the coupled-mode equations can be solved analytically. For a gradual taper profile, the solution was expressed in orders of a perturbation matrix $\overline{\bar{e}}$. The solution is valid for an arbitrary profile shape.

Analytic solutions for particular tapered coupler design were obtained as follows. The two-mode forward coupling was explicitly solved for a $\mathrm{TE}_{10}^{\text {fast }}$-to- $\mathrm{EH}_{10}^{\text {slow }}$ converter with a simple linear taper profile. The unwanted $\mathrm{EH}_{11}$ power was found to be $-12-\mathrm{dB}$ below the input $\mathrm{TE}_{10}$ power over the 30 to $40-\mathrm{GHz}$ range. The $\mathrm{EH}_{12}$ and $\mathrm{HE}_{11}$ modes were $-45 \mathrm{~dB}$ below the input level. By examining the coupling coefficient, the profile was then improved by a three-piece linear taper to reduce the unwanted power in the $\mathrm{EH}_{11}$ mode to less than $-27 \mathrm{~dB}$ at $35 \mathrm{GHz}$, and no more than $-20 \mathrm{~dB}$ in the 32 to $40 \mathrm{GHz}$ range.

\section{ACKNOWLEDGMENT}

The authors wish to acknowledge helpful discussions with J. Joe, B. McVey, and J. Walsh.

\section{REFERENCES}

[1] D. J. Radack, J.H. Booske, Y. Carmel, and W. W. Destler, "Wiggler focused relativistic sheet beam propagation in a planar free-electron laser configuration," Appl. Phys. Lett., vol. 55, no. 20, pp. 2069-2071, Nov. 1989.

[2] J. H. Booske et al., "Design of high-average-power near-millimeter free electron laser oscillators using short-period wigglers and sheet electron beams," IEEE Trans. Plasma Sci., vol. 18, pp. 399-415, June 1990.

[3] A. Ferendeci, "Synthesis and design of sheet beam for gyrotrons," presented at the 31th APS, Anaheim, Los Angeles, Nov. 13-17 1989. 
[4] M. E. Read, et al., "Design and testing of a sheet beam electron gun for a quasi-optical gyrotron," in Conf. Dig., 14th Int. Conf. on Infrared and Millimeter Waves (Wurzburg, Germany), 1989, vol. 1240, pp. 79-80.

[5] E. Garate, C. H. Shaughnessy, and J. Walsh, in IEEE J. Quant. Electron, vol. QE-23, p. 1627, 1987.

[6] E. Garate, C. Shaughnessy, B. Johnson, J. Walsh, and S. Moustaizis, in Nucl. Instrum., Method., vol. A259, p. 125, 1987.

[7] H.C. Chen, "General theory of the diocotron instability of a relativistic electron beam," IEEE Trans. Nucl. Sci., vol. NS-32, pp. 2380-2382, Oct. 1985.

[8] T.M. Antonsen, Jr. and E. Ott, "Velocity shear driven instabilities of an unneutralized electron beam," Phys. Fluids, vol. 18, no. 9, pp. 1197-1208, Sept. 1975 .

[9] R. C. Davidson, K. T. Tsang, and H. S. Hum, "Diocotron instability for intense relativistic non-neutral electron flow in planar diode geometry," Phys. Fluids, vol. 31, pp. 1727-1737, 1988.

[10]' V.G. Leiman, A. A. Tevryukov, A.P. Ovchinnikov, and G. N. Freiberg "Ribbon-shaped electron beams for plasma-chemistry reactors" Sov. $J$ Plasma Phys., vol. 4, p. 377, 1978.

[11] J. Walsh and J. Murphy, "Tunable Cerenkov lasers," IEEE J. Quant. Electron., vol. 18, no. 8, pp. 1259-1263, Aug. 1982

[12] W. Case, "Basic theory of Cerenkov radiations," in High-Power Microwave Sources, V. Granastein and I. Alexeff Eds. Boston: Artech, 1987.

[13] T. Shiozawa and H. Kondo, "Mode analysis of an open-boundary Cerenkov laser in the collective regime," IEEE J. Quant. Electron., vol. 23, no. 9, pp. 1633-1641, Sept. 1987

[14] J. D. Lawson, The Physics of Charged Particles. Oxford, UK: Clarendon, 1988, chap. 6, p. 293; (1988); see also, J. Joe, S.F. Chang, J. Scharer, and J. Booske, "Linear analysis of sheet beam planar grating and dielectric Cerenkov maser amplifiers," Microwave Opt. Techn. Lett., vol. 4 , No. 11 , p. 443 , Oct. 1991

[15] H.P. Freund, "Nonlinear theory of slow-wave ubitron/free-electron masers," Bull. Amer. Phys. Soc., vol. 35, no. 9, p. 2143, 1990.

[16] W. B. Case, R. D. Kaplan, J.E. Golub, and J.E. Walsh, "Space-chargeCerenkov and cyclotron-Cerenkov instabilities in an electron beam dielectric system," J. Appl. Phys., vol. 55, no. 7, pp. 2651-2658, Apr. 1984.

[17] T.H. Kuo and A.T. Lin, "Cyclotron-Cherenkov and Cherenkov instabilities," IEEE Trans. Plasma Sci., vol. 18, pp. 513-517, June 1990.

[18] H. Freund and A. Ganguly, in Phys. Fluids, vol. B2, p. 2506, 1990.

$19]$ R. F. Harrington, Time-Harmonic Electromagnetic Fields. New York: McGraw-Hill, 1961, chap. 4.

[20] S. F. Chang, "Analysis of dielectric Cerenkov masers and wiggler freeelectron lasers in the millimeter wavelength range," $\mathrm{Ph} . \mathrm{D}$. thesis, Univ. Wisconsin-Madison, chap. 2, Aug. 1991.

[21] L. R. Barnett et al., "Absolute instability competition and suppression in a millimeter-wave gyrotron traveling-wave tube," Phys. Rev. Lett., vol. 63, pp. 1062-1065, Sept. 1989.

[22] P.E. Latham, B. Levush, and T. M. Antonsen, Jr., "Harmonic operation of free electron laser," Phys. Rev. Lett., vol. 66, no. 11, pp. 1442-1445, 1991.

[23] R. E. Collin, Field Theory of Guided Waves, 2nd Ed. New York: IEEE 1991.

[24] C.H. Tang, "Optimization of waveguide tapers capable of multimode propagation," IRE Trans. Microwave Theory Tech., vol. MTT-9, pp. $442-452$, Sept. 1961 .

[25] R.P. Hecken, "A near-optimum matching section without discontinuities," IEEE Trans. Microwave Theory Tech., vol. 20, pp. 734-739, Nov. 1972.

[26] R.P. Hecken and A. Anuff, "On the optimum design of tapered waveguide transitions," IEEE Trans. Microwave Theory Tech., vol. 21 pp. 374-380, June 1973 .

[27] W.G. Lawson, "Theoretical evaluation of nonlinear tapers for high-power gyrotron," IEEE Trans. Microwave Theory Tech., vol. 38, pp. 1617-1622, Nov. 1990.

[28] R. A. Schill and S. R. Seshadri, "Optimization of a bumpy cylindrical waveguide mode converter," Int. J. Infrared Millimeter Waves, vol. 7, no. 8 , pp. $1129-1167,1986$.

[29] M. Buckley and R. Vernon, "Compact quasi-periodic and aperiodic $\mathrm{TE}_{\text {on }}$ mode converters in overmoded circular waveguides for use with gyrotrons," IEEE Trans. Microwave Theory Tech., vol. 38, pp. 712-721, June 1990.

[30] G. H. Luo, D. A. Casper, and R. J. Vernon, "Design of a single-period 8.6-GHz TM01-TE11 serpentine mode converter and TM01 and TE1 bends in a moderately overmoded circular waveguide," presented at the 15th Int. Conf. on Infrared and Millimeter Waves, Florida, Dec. 10-14, 1990.

[31] R. L. Kyhl and H.F. Webster, "Breakup of hollow cylindrical electron beams," IRE Trans. Electron Devices, pp. 172-183, Oct. 1956.

[32] J. Pierce, "Instability of hollow beams," IRE Trans. Electron Devices, pp. 184-189, Oct. 1956

[33] R.H. Levy, "Diocotron instability in a cylindrical geometry," Phys. Fluids, vol. 8 , no. 7 , pp. $1288-1295$, July 1965 .

[34] H.S. Uhm and J.G. Siambis, "Diocotron instability of a relativistic hollow electron beam," Phys. Fluids, vol. 22, no. 12, pp. 2377-2381, Dec. 1979

[35] V. A. Lebedev, I.N. Meshkov, and A. N. Sharapa, "Dynamics of an intense ribbon electron beam in its self-magnetic field," Sov. Phys. -Tech. Phys., vol. 24, no. 4, pp. 421-424, Apr. 1979.

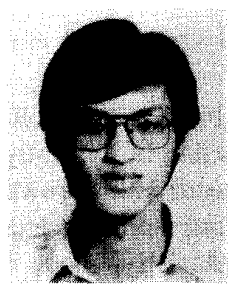

Sheng-Fuh R. Chang was born in Taiwan, Republic of China, in 1960. He received the B.S. and M.S degrees in communication engineering from the National Chiao-Tung University in 1982 and 1984 respectively.

He has been working towards the Ph.D. degree in electrical and computer engineering and is a member of the Center for Plasma Theory and Computation a the University of Wisconsin, Madison. His research interests are in theoretical and computational investigations in the area of coherent radiation sources plasma waves, and microstrip circuits.

Mr. Chang is a member of Phi Tau Phi.

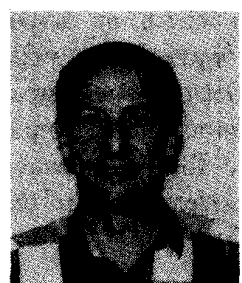

John E. Scharer received the B.S., M.S., and Ph.D. degrees in electrical engineering from the University of California, Berkeley, in plasma physics.

$\mathrm{He}$ is a Professor with the Department of Electrical and Computer Engineering, University of Wisconsin, Madison. He has spent research sabatticals at the CEA Fontenay-aux-Roses, France, in 1970 and at the JET tokamak at Culham, England in 1983. He has been active with graduate students and scientists in theoretical, computational, and experimental research in the area of linear and nonlinear plasma waves and heating of fusion plasmas and lasers. He has also taught and recently started research in the area of free-electron lasers and coherent sources of radiation.

Dr. Scharer is a member of Tau Beta Pi, the American Physical Society, and the Center of Plasma Theory and Computation at the University of Wisconsin.

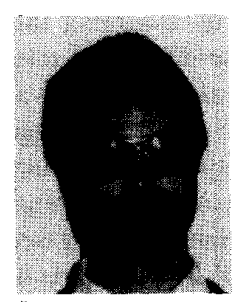

John H. Booske (S'82-M'89) received the Ph.D. degree in nuclear engineering from the University of Michigan, Ann Arbor, in 1985. Topics of graduate research included high-power $\mathrm{CO}_{2}$ lasers and electron-cyclotron resonant heating and cyclotron emissions from magnetically confined hot electron plasmas.

In 1985 he began work in the Laboratory for Plasma Research, University of Maryland, College Park, as a Research Associate, studying hot ion microinstabilities in magnetic mirror plasmas. $\mathrm{He}$ subsequently joined the Research and Graduate faculties at the University of Maryland, with responsibility for research in millimeter-wave free electron lasers. He is currently a faculty member in the Department of Electrical and Computer Engineering, University of Wisconsin, Madison. His research interests include free electron and plasma radiation sources, plasma heating and wave interactions, and millimeter-wave/dielectric interactions. 